[Agr. Biol. Chem., Vol. 26, No. 10, p. 699 704, 1962]

\title{
Studies on the Flavonoids in Tea Leaves
}

\author{
Part VI.* On the Myricetin-glycoside \\ By Yoshinori Takino**, Hiroshi Imagawa** and Hiroyuki Yoshida*** \\ ** Department of Agricultural Chemistry, Faculty of Agriculture, Tokyo University of Education, \\ Tokyo; *** Saitama Prefectural Tea Experimental Station, Saitama \\ Received June 14, 1962
}

\begin{abstract}
Yellow needle crystals, $\mathrm{C}_{21} \mathrm{H}_{20} \mathrm{O}_{13} \cdot \mathrm{H}_{2} \mathrm{O}$ have been isolated from tea leaves. The crystals yield myricetin (hexaacetate, m.p. $211 \sim 212^{\circ} \mathrm{C}$ ), glucose and galactose on hydrolysis. As analytical data indicate the molecular ratio of myricetin to the sugars to be $1: 1$ and the only bonding position of the sugars to be position 3 of the aglycone, the crystals are concluded to consist of two kinds of glycosides, namely myricetin-3-glucoside and myricetin-3-galactoside.
\end{abstract}

On the flavonoid constituents in green tea leaves, three natural pigments were isolated previously in this laboratory ${ }^{1)}$. One of them was identified as rutin, but the others were new flavonoids. Detailed elucidation of their chemical constitutions led to the conclusion that they are glycosides of kaempferol and quercetin, which contain a trisaccharide composed of two moles of glucose and one mole of rhamnose at position 3 of each aglycone. Recently the authors proposed to give them new names, kaempfetrin and quertrin respectively ${ }^{2)}$. Oshima and Nakabayashi ${ }^{3)}$ also pointed out the same glycosides to be contained in the leaves of Assam-variety together with several related flavonoids, such as astragalin, quercitrin, isoquercitrin, and kaempferol-rhamnoglucoside, etc.

On the other hand, in testing the methanolic extract of the leaves of Yabukita-variety by two-way chromatography in our laboratory, another unidentified spot of flavonoid was

* Part VI of a series entitled, "Biochemical Studies on Tea Leaves". 1) Y. Takino, H. Imagawa and H. Yoshida, J. Agr. Chem. Soc. Japan, 27,150 (1953); 28, 182, 186, 190 (1954).

2) Y. Takino, H. Imagawa and H. Yoshida, ibid., in press.

3) Y. Oshima and T. Nakabayashi, ibid., 27, 274, 754, 756, 759 (1953). detected. After laborious treatment of purification it was isolated in yellow needles, the aglycone of which was identified as myricetin, and glucose was detected in the hydrolysate of the crystals ${ }^{4}$. Roberts et al.5) have also reported the presence of myricetin-glucoside and -rhamnoglucoside in Indian tea leaves, but their components probably were not obtained in crystals. However, as the search on the constitution of our crystals progresses, some additional informations have been obtained.

\section{EXPERIMENTAL}

Samples. Fresh tea leaves of several varieties were applied; viz. Yabukita, Indo and Benihomare, growing in the field of the Saitama Prefectural Tea Experimental Station. Yabukita is a variety which is recommended for manufacturing superior green tea. The others are varieties selected amongst tea plants grown from the seeds which had been introduced from India and known to be suitable for manufacturing black tea.

Paper Chromatography. Plucked leaves (shoots

4) Y. Takino, H. Imagawa and H. Yoshida, presented at the 151 st Meeting of the Kanto-branch of the Agricultural Chemical Society of Japan, Tokyo, December 18, 1954.

5) E.A.H. Roberts, R.A. Cartwright and D.J. Wood, J. Sci. Food Agric., 7, 637 (1956). 


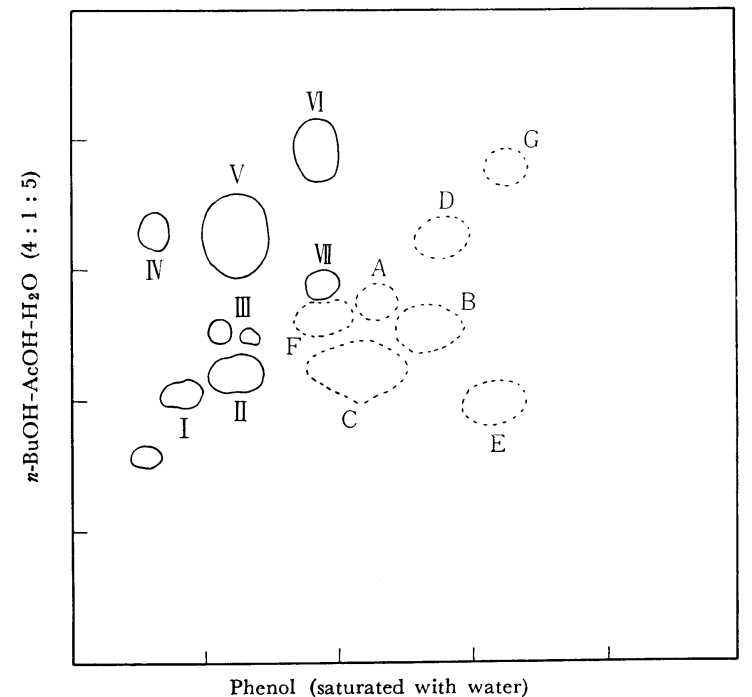

FIG. 1. Principal Polyphenols Occurring in Tea Leaves.

Catechins (blue or violet)*: Flavonoids (green or brown)*

$$
\begin{array}{ll}
\text { I. Theogallin } & \text { A. Rutin } \\
\text { II. } & \text { C. Quenpin }
\end{array}
$$

II. (-)-epiGallocatechin $\quad$ B. Kaempfetrin

III. ( \pm -Gallocatechin $\quad$ C. Quertrin

IV. Gallic acid D. Isoquercitrin

V. (-)-epiGallocatechin $\quad$ E. unidentified** gallate

VI. (-)-epiCatechin gallate F. Myricetin-glycoside

VII. (-)-epiCatechin G. Astragalin

* Color reaction with $1 \%$ solution of aqueous iron alum.

** This spot seems to be kaempferol-glycoside by our preliminary test.

consisting of three leaves and the terminal apex) were steamed, dried and ground. Five hundred miligrams of the powdered leaves were extracted with benzene several times to remove chlorophyll. The residue was then extracted repeatedly with successive $10 \mathrm{ml}$ portions of methanol. The methanolic extract was made up to $50 \mathrm{ml}, 1 \mathrm{ml}$ portion of which was concentrated and subjected to two-way chromatography. Fig. 1 shows the chromatogram of the polyphenols occurring in an appreciable amount in tea leaves. Some minor constituents are omitted.

Of these polyphenols, unidentified spot $\mathrm{F}$ was found not only in the leaves of Yabukita, but also in Indo and Benihomare in a considerable amount. Therefore it is supposed to be a common constituent in tea leaves. As it appears brownish under ultraviolet light, and gives a temporary yellow color with ammonia vapor and permanent yellow color when sprayed with aqueous basic lead acetate or aluminum chloride, and dark greenish blue with aqueous ferric alum, it may be supposed to be a flavonol glycoside. In order to characterize this constituent its isolation was tried.

Isolation of the Glycoside. Powdered green tea manufactured from the leaves of Yabukita was extracted twice with hot water. The combined turbid extracts were then treated with $10 \%$ aqueous solution of lead acetate until the supernatant liquid became fairly transparent. After removal of the precipitated mass by filtration under suction, fractional precipitation with lead acetate was continued until no more precipitation was made. Substance $\mathbf{F}$ was included mostly in the earlier precipitate together with a greater amount of catechin gallates and some oxidation products. This precipitate was then suspended in methanol and decomposed by hydrogen sulfide. The filtrate freed from lead sulfide was concentrated in vacuo and the residue was dissolved in water. The aqueous solution was shaken first with chloroform and then with ether to remove caffeine and some other impurities. Addition of sodium chloride to the solution produced dark brown glutinous precipitate. Filtrate and washings were combined, extracted with ethyl acetate, and the extracts were concentrated under reduced pressure.

Further fractionation was carried out by partition chromatography using silica gel column as described previously ${ }^{1)}$, but this time ethyl acetate was used as developing solvent. As the partition progresses, catechin gallates ran down most rapidly through the column and was followed by free catechins. Then three separate yellow bands appeared on the column. They are isoquercitrin, pigment $\mathbf{F}$ and rutin, in order. The fractions corresponding to the second yellow band were collected after repeating the procedure several times; and the solvent was distilled off. On cooling the concentrated aqueous solution, resinous mass appeared and settled at the bottom of the vessel. The supernatant liquid was then poured into another vessel. Having been kept in an ice box, yellow needles crystallized out. They were collected after several days and recrystallized several times from aqueous methanol. Yield: about $150 \mathrm{mg}$ from $3.5 \mathrm{~kg}$ of green tea. M.p. $224 \sim 225^{\circ} \mathrm{C}$ (decomp.).

Reduction with magnesium or zinc and conc. hydrochloric acid in methanol gives a red color. The glycoside is precipitated from aqueous solution by lead acetate at lower $\mathrm{pH}$ as compared with other known 


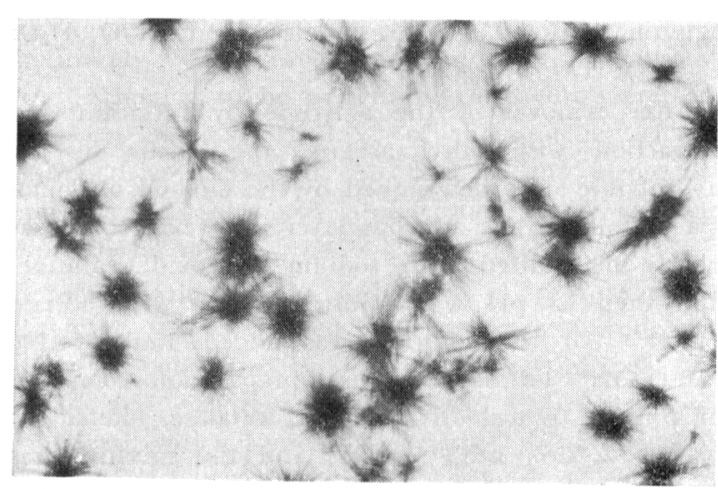

FIG. 2. Crystals of the Myricetin-glycoside (from water).

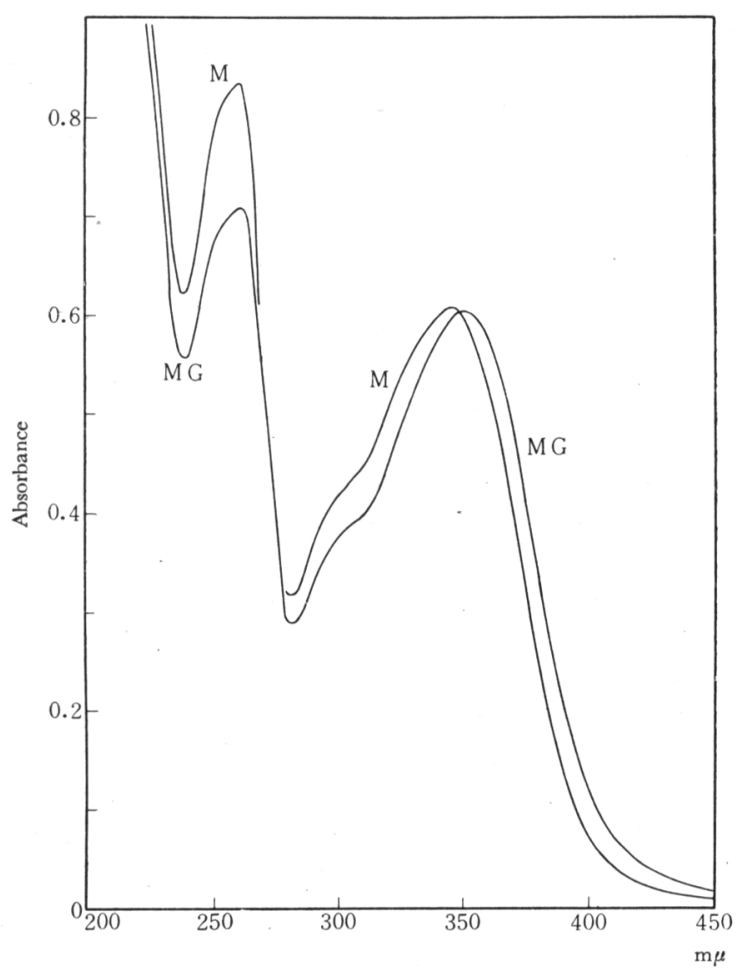

FIG. 3. Absorption Spectra of the Myricetin-glycoside and Myricitrin in Aqueous Solution.

MG : Myricetin-glycoside (anhydrous, $18.029 \mathrm{mg} / \mathrm{l}$ ).

${ }^{\lambda} \max 262 \mathrm{~m} \mu(\varepsilon, 18,800), 350 \mathrm{~m} \mu(\varepsilon, 16,000)$. M: Myricitrin (anhydrous, $18.803 \mathrm{mg} / \mathrm{l}$ ). Observed in $1-\mathrm{cm}$ cells.

tea leaf flavonoids. Solubility: slightly soluble in water; soluble in hot water, butanol and ethyl acetate; very soluble in methanol and ethanol; but insoluble

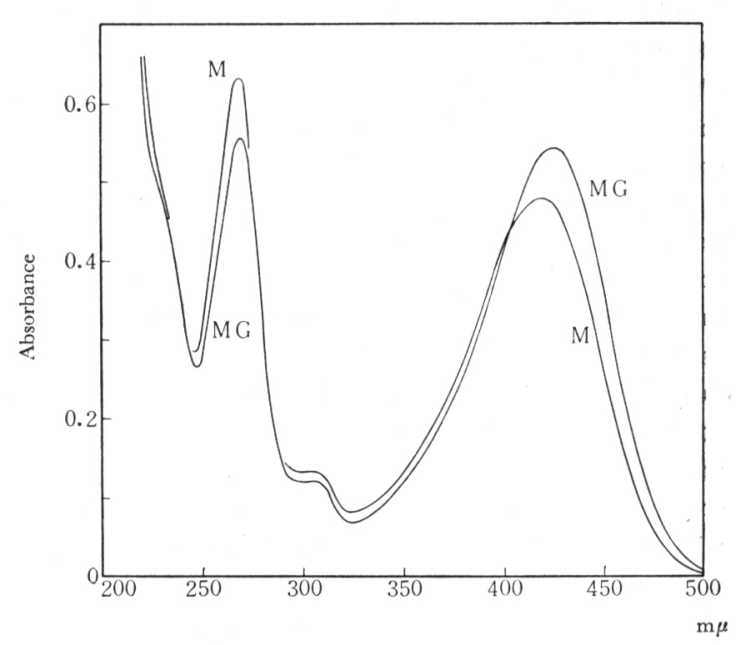

FIG. 4. Absorption Spectra of the Myricetin-glycoside and Myricitrin in $0.4 \%$ Aqueous Aluminum Chloride Solution.

MG : Myricetin-glycoside (anhydrous, $10.817 \mathrm{mg} / \mathrm{l}$ ). $\lambda_{\max } 269 \mathrm{~m} \mu(\varepsilon, 24,800), 425 \mathrm{~m} \mu(\varepsilon, 24,100)$. $\mathrm{M}$ : Myricitrin (anhydrous, $11.282 \mathrm{mg} / \mathrm{l}$ ). Observed in $1-\mathrm{cm}$ cells.

in ether and chloroform.

The glycoside, which was kept for a long time in a calcium chloride desiccator, lost $3.62 \%$ of its weight when heated in vacuo over phosphorous pentoxide at $115^{\circ} \mathrm{C}$. Calcd. for $\mathrm{C}_{21} \mathrm{H}_{20} \mathrm{O}_{13} \cdot \mathrm{H}_{2} \mathrm{O}: \mathrm{H}_{2} \mathrm{O}, 3.61 \%$. Analytical data of the anhydrous substance was as follows: Found: $\mathrm{C}, 52.49 ; \mathrm{H}, 4.23$. Calcd. for $\mathrm{C}_{21} \mathrm{H}_{20} \mathrm{O}_{13}$ : $\mathrm{C}$, $52.50 ; \mathrm{H}, 4.17 \%$. The UV-spectra of this substance in water bear a striking resemblance to that of myricitrin (myricetin-3-rhamnoside) as shown in Figs. 3 and 4.

Hydrolysis of the Glycoside. The glycoside was hydrolyzed in $5 \%$ aqueous sulfuric acid solution on a boiling water bath for two hours. The resulting crystals were separated the next day and washed. The mother liquor and washings of the hydrolysis were neutralized with barium carbonate. After removal of the precipitated barium sulfate, the filtrate was concentrated and submitted to the identification of sugar components.

A small portion of the aglycone was dissolved in methanol and proved by paper chromatography, using $n$-butanol-acetic acid-water $(4: 1: 5, \mathrm{v} / \mathrm{v})$ and phenol saturated with water as developing solvents, and the spots were observed under UV-light, alone or under 
humid condition of ammonia vapor. They were also sprayed with aqueous basic lead acetate or ferric alum. The sugars in the filtrate were also proved by the same method, but their spots were detected by spraying aniline hydrogen phthalate. The results are summarized in Table I, from which it may be sup. posed that the glycoside contains myricetin as aglycone, and two kinds of hexoses (probably glucose and galactose) as sugar components. In order to ascertain the presence of these hexoses more exactly, .two-way chromatographic method was applied repeatedly, using several kinds of solvent systems. Phenol was the most effective; for instance, when the sample spotted on the paper was run first twice repeatedly with phenol in the same direction and then once with phenol in another direction, there appeared two separate spots, showing apparently near intensity of color. Each of them was intensified by mixing the sample with an authentic specimen of glucose or galactose respectively. This observation indicates that both glucose and galactose are the most probable sugar components of the glycoside.

TABLE I. COMPARISON OF HYDROLYZED PRODUCTS OF THE GLYCOSIDE WITH VARIOUS SPECIMENS BY MEANS OF PAPER CHROMATOGRAPHY

Aglycone :

\begin{tabular}{lccc} 
Substance & \multicolumn{3}{c}{$R_{F}$-Value } \\
$\begin{array}{l}\text { Aglycone of the } \\
\text { glycoside }\end{array}$ & 0.47 & 0.17 & 0.26 \\
Kaempferol & 0.86 & 0.68 & 0.53 \\
Quercetin & 0.71 & 0.37 & 0.42 \\
Myricetin & 0.47 & 0.17 & 0.26 \\
Sugar : & & & Acetic acidc) \\
Substance & Butanola) & Phenolb) & Collidineb) \\
Sugars of the & 0.17 & 0.53 & 0.28 \\
glycoside & 0.18 & 0.48 & 0.48 \\
Glucose & 0.16 & 0.53 & 0.26 \\
Galactose & 0.22 & 0.55 & 0.40 \\
Mannose & &
\end{tabular}

a) $n$-Butanol-acetic acid-water $(4: 1: 5)$. b) Saturated with water. c) Acetic acid-water $(6: 4)$.

Molecular Ratio of the Sugars to the Aglycone. Hydrolysis of $69.5 \mathrm{mg}$ of the crystals yielded $44.6 \mathrm{mg}$ of aglycone, which corresponds to $64.2 \%$ of the original material. If the glycoside consists of each one mole of myricetin and hexose, the calculated yield of the aglycone must be as follows: Calcd. for $\mathrm{C}_{21} \mathrm{H}_{20} \mathrm{O}_{13} \cdot \mathrm{H}_{2} \mathrm{O}$ : $\mathrm{C}_{15} \mathrm{H}_{10} \mathrm{O}_{8} \cdot \mathrm{H}_{2} \mathrm{O}, 67.5 \%$.

After removal of the aglycone by filtration and extraction with ethyl acetate, the sugars in the hydrolyzate were determined by the method of Somogyi as follows: the aqueous layer after extraction was nearly neutralized with sodium hydroxide solution, and then its $\mathrm{pH}$ was adjusted to 5.8 with acetate buffer. To the solution was added $1 \mathrm{ml}$ of $1 \%$ "DEOXIN" solution (enzyme preparation composed of purified glucose oxidase and catalase, offered by Nagase \& Co., Ltd., Japan), and the mixture was incubated at $35^{\circ} \mathrm{C}$ for four hours, while bubbling air through the system. As the enzyme oxidizes only glucose specifically and almost completely (more than $98 \%$ ) but has no effect on other sugars, the difference of reducing values of the mixture before and after incubation corresponds to the content of glucose, and the value after incubation to that of galactose. Thus, $10.7 \mathrm{mg}$ of glucose and $14.2 \mathrm{mg}$ of galactose are estimated, and therefore the total sugars amount to $24.9 \mathrm{mg}$, which corresponds to $35.8 \%$ of the original material. Calcd. for $\mathrm{C}_{21} \mathrm{H}_{20} \mathrm{O}_{13} \cdot \mathrm{H}_{2} \mathrm{O}: \mathrm{C}_{6} \mathrm{H}_{12} \mathrm{O}_{6}, 36.1 \%$.

If the glycoside were to consist of one mole of aglycone and two moles of hexoses, the yield of the aglycone should be $35.0 \mathrm{mg}(50.4 \%)$ and that of sugars $37.5 \mathrm{mg}(54.0 \%)$. The experimental results do not support this assumption and the ratio $1: 1$ seems to be most probable. However, considering that some errors might have been unavoidable in the yield of aglycone, we carried out another accurate micro-determination of the ratio, as follows: $4.072 \mathrm{mg}$ of the glycoside was hydrolyzed in $5 \%$ aqueous sulfuric acid solution on a boiling water bath for an hour. The precipitated aglycone was collected on a small glass-filter and the filtrate was neutralized with sodium hydroxide solution. After the aglycone on the filter was dissolved in $30 \mathrm{ml}$ of ethanol and combined with the filtrate, they were made up to $100 \mathrm{ml}$ exactly with water. To an aliquot $(1.0 \mathrm{ml})$ of the solution were added $2.0 \mathrm{ml}$ of $1 \%$ aqueous aluminum chloride solution and $2.0 \mathrm{ml}$ of water. The yellow aluminum complex of myricetin has an absorption maximum at $438 \mathrm{~m}_{\mu}$. The absorbance of the solution was determined with a spectrophotometer at $438 \mathrm{~m} \mu$, using $1-\mathrm{cm}$ cells, and the content of myricetin monohydrate was calculated from the calibration curve, which was standardized by the use of an authentic specimen of 
myricetin monhydrate and proved to obey Beer's law accurately. Thus $2.705 \mathrm{mg}$ of myricetin monohydrate was estimated to be present in the hydrolyzate, and this corresponds to $66.4 \%$ of the starting sample. From these facts and the data of the elementary analysis this substance is concluded to be a monohexoside of myricetin.

Identification of the Aglycone. A portion of the aglycone was acetylated with acetic anhydride and pyridine, and the product was recrystallized from methanol. Colorless needles, m.p. $211 \sim 212^{\circ} \mathrm{C}$ were obtained, which showed no melting point depression on mixing with an authentic specimen of hexaacetyl myricetin. Anal. Found: C, 56.62; H, 4.13. Calcd. for $\mathrm{C}_{27} \mathrm{H}_{22} \mathrm{O}_{14}: \mathrm{C}, 56.84 ; \mathrm{H}, 3.86 \%$. The aglycone of the glycoside is therfore concluded to be myricetin.

Identification Tof the Sugars. Concerning the sugar components glucose and galactose were detected by the method of paper chromatography as above. The presence of glucose is also supported by the fact that about $43 \%$ of the sugars in the hydrolyzate of the glycoside was oxidized by the glucose oxidase as shown in the above experiment. Then the remaining sugar was identified to be galactose as follows: the solution after oxidation of glucose $(4 \mathrm{ml})$ was 'concentrated and heated with $20 \mathrm{mg}$ of phenylhydrazine hydrochloride in the presence of $40 \mathrm{mg}$ of sodium acetate for about an hour. Small amount of yellow mass was preciptated. Crystallization from ethanol yielded yellow needles, m.p. $192^{\circ} \mathrm{C}$ (decomp.), which showed no melting point depression on mixing with an authentic specimen of phenyl-D-galactosazone.

Position of the Sugars. The glycoside shows positive reaction when it is treated with the reagent magnesium and hydrochloric acid, and also with zinc and hydrochloric acid. According to the description of Shimizu ${ }^{6}$, the fact suggests that the position of the sugars linking to the aglycone may be position 3 . To obtain more definite evidence the following procedure was carried out:

To $48.6 \mathrm{mg}$ of the glycoside suspended in $30 \mathrm{ml}$ of dry acetone were added $4 \mathrm{~g}$ of potassium carbonate and $1.7 \mathrm{ml}$ of dimethyl sulfate. After seven hours boiling on a water bath, the solution gave no further color reaction with ferric chloride. Water was now added and the acetone was distilled off. The residue was hydrolyzed with $3 \%$ sulfuric acid. The resulting

6) M. Shimizu, J. Pharm. Soc. Japan, 72, 338 (1952). precipitate was collected after overnight standing, and recrystallized two times from methanol. The pale yellow needles, m.p. $225^{\circ} \mathrm{C}$ were obtained. Anal. Found: $\mathrm{C}, 61.71 ; \mathrm{H}$, 5.11. Calcd. for $\mathrm{C}_{20} \mathrm{H}_{20} \mathrm{O}_{8}$ : C, $61.86 ; \mathrm{H}, 5.15 \%$. The methylated aglycone seems to be $5,7,3^{\prime}, 4^{\prime}, 5^{\prime}$-pentamethyl myricetin. For comparison, the same compound was prepared by the hydrolysis of completely methylated myricitrin (from the bark of Myrica rubra Sieb. et Zucc.). Because the mixed melting point of these compounds showed no depression, the methylated aglycone is without doubt 3hydroxy-5,7,3',4',5'-pentamethoxyflavone. It is, therefore, concluded that the sugars are linking to the hydroxyl group at position 3 of myricetin.

\section{DISCUSSION}

From the evidence in this paper it was established that the pigment now obtained in crystals is composed of myricetin, glucose and galactose, but the noticeable fact is that the ratio of aglycone to sugar is $1: 1$. But two kinds of hexoses were found in the crystalline preparation in spite of the above ratio, it must, therefore, be concluded that the crystalline preparation consists of two kinds of glycosides, each aglycone of which is the same myricetin and each sugar is combined to other molecule of myricetin separately to form myricetin-glucoside and -galactoside. Properties of these glycosides are so similar that they seem to be scarcely separated from each other by any of the usual solvents and give the same single spot on the chromatograms. Roberts et al.5) have described analogous relation between 3-galactoside and 3-glucoside of kaempferol as their own experience and also Siegelman's private communication. Thus from tea leaves two kinds of glycosides of myricetin must have been isolated in mixed crystals, which seem to consist of about $43 \%$ of glucoside and $57 \%$ of galactoside according to the analysis of sugars. Attempts to separate them into two components are now under study.

The discovery of this myricetin-galactoside 
seems to have given us new information on the flavonoids of tea leaves and a new problem especially in their biochemical respect, because the known flavonoids in tea leaves contain only glucose or rhamnose or both of them as sugar component, and galactoside of kaempferol or quercetin have not yet been found in tea leaves. Concerning the sugars in tea leaves, free or in combination, Torii and Kanazawa ${ }^{7}$ ) have reported the detection of them by paper chromatographic method. They fractionated the constituents of tea leaves into several fractions. One of them, which was obtained by extraction of tea leaves with ethanol and precipitation with neutral lead acetate, gave spots of glucose, galactose, arabinose, xylose, and rhamnose after hydrolysis with aqueous sulfuric acid. They supposed that glucose and rhamnose are the components of flavonoids, whereas glucose, galactose and arabinose are that of saponins. But the glycosides in our paper are thought to be included in the above fraction on such a treatment of fractionation, galactose detected in this fraction by Torii and Kanazawa seems, therefore, to have been derived at

7) H. Torii and J. Kanazawa, J. Agr. Chem. Soc. Japan, 28, 34 (1954). least partly from flavonoid, myricetin-galactoside.

Concerning the glycosides of myricetin, some are known in nature,-besides myricitrin found long before in the barks of Myrica rubra ${ }^{8}$-for instance, myricetin glycoside in the flower of Acacia leuciphloea ${ }^{9}$, myricetin3-glucoside in the pollen of Acacia dealbata ${ }^{\mathbf{1 0})}$, and myricetin-3-digalactoside in the birch leaves ${ }^{11)}$. Roberts et al.5) have pointed out the presence of myricetin-rhamnoglucoside in tea leaves from North East India. Now the detection of myricetin-3-galactoside in tea leaves seems to be a new contribution to the flavonoids chemistry.

Acknowledgement. The authors wish to express their sincere thanks to Prof. R. Yamamoto, Tokyo Agricultural College and Prof. Y. Oshima, Kyushu University for their kind advice and encouragement throughout this work.

8) A.G. Perkin and J.J. Hummel, J. Chem. Soc. London, 69, 1287 (1896).

9) S.K. Mnkerjee and V.V.S. Murty, J. Sci. Ind. Research, 11 B125 (1952); C.A., 47, 3524 (1953).

10) A. Spada and R. Cameroni, Gazz. Chim. Ital., 86, 965 (1956) C.A., 52, 12092 (1958).

11) L. Hörhammer, H. Wagner and R. Luck, Arch. Phaım., 290 338 (1957); C.A., 51, 17099 (1957). 